\title{
Guest Editorial: Computer Science Symposium in Russia
}

\author{
Lev Beklemishev ${ }^{1,2,3}$
}

Published online: 9 May 2017

(C) Springer Science+Business Media New York 2017

This special issue is devoted to a broad range of theoretical topics in Computer Science, including computational complexity theory, formal grammars, algorithms, automata, Kolmogorov complexity, and quantum complexity. If there is a common theme among this diversity, it is the one of complexity, a major concern for each and every contribution to this special issue.

The paper Bounds in Ontology-Based Data Access via Circuit Complexity by Vladimir Podolskii is a survey of recent results on the size of query rewriting and on methods for proving lower and upper bounds for it based on Boolean circuit complexity.

The authors of the paper Equations Over Free Inverse Monoids with Idempotent Variables by Volker Diekert, Florent Martin, Géraud Sénizergues, and Pedro V. Silva introduce the notion of idempotent variables for studying equations in free monoids. They provide upper and lower bounds for the complexity of the problem of solvability of systems of equations in idempotent variables in free inverse monoids. Their results also apply to families of equations where no decidability has been previously known.

The paper Some Properties of Antistochastic Strings by Alexey Milovanov deals with algorithmic statistics, a part of Kolmogorov complexity theory. The author introduces the notion of antistochastic string and demonstrates, among other things, that such strings satisfy the following property: if an antistochastic string $x$ has

\section{Lev Beklemishev}

lbek1@yandex.ru

1 Steklov Mathematical Institute of the Russian Academy of Sciences, Moscow, Russia

2 National Research University Higher School of Economics, Moscow, Russia

3 Moscow M.V. Lomonosov State University, Moscow, Russia 
complexity $k$, then any $k$ bits of information about $x$ are enough to reconstruct $x$ (with logarithmic advice).

In the paper Rewriting higher order stack trees by Vincent Penelle the author introduces the notion of stack trees, that is, trees whose nodes are labelled by higher-order stacks, and defines the corresponding class of higher-order ground tree rewriting systems. He shows that these graphs retain the decidability properties of ground tree rewriting graphs and generalise the pushdown hierarchy of graphs.

The paper Generalized LR Parsing Algorithm for Grammars with One-Sided Contexts by Mikhail Barash and Alexander Okhotin generalizes the efficient LR parsing algorithm for context-free grammars to the wider class of 'grammars with left contexts'. The resulting algorithm is applicable to any grammar with left contexts and has the same worst-case cubic-time performance as for the context-free grammars.

In the paper Optimizing binary heaps by Stefan Edelkamp, Amr Elmasry, Jyrki Katajainen the authors study efficient implementations of the priority queue data structure via binary heaps. They propose a variant of a binary heap that operates in-place, executes minimum and insert operations in $O(1)$ worst-case time, and extract-min in $O(\lg n)$ worst-case time while involving at most $\lg n+O(1)$ element comparisons. These bounds surpass the lower bounds known for binary heaps and answer a long-standing question in this area.

The paper On Compiling Structured CNFs to OBDDs by Simone Bova and Friedrich Slivovsky contains new results on the size of OBDD representations of structurally characterized classes of CNF formulas. First, the authors prove that variable convex formulas (that is, formulas with incidence graphs that are convex with respect to the set of variables) have polynomial OBDD size. Second, they prove an exponential lower bound on the OBDD size of a family of CNF formulas with incidence graphs of bounded degree.

The paper On the Satisfiability of Quantum Circuits of Small Treewidth by Mateus de Oliveira Oliveira is dedicated to the study of quantum analogs of certain NP-hard optimization problems restricted to structures of constant treewidth and to proving appropriate complexity bounds.

The special issue was initiated by the 10th International Computer Science Symposium in Russia (CSR 2015) held in Listvyanka (lake Baikal, Irkutsk region) in July 2015. The journal articles were selected among the top contributions to the conference, including the three winning papers of the best paper prize. The conference papers were substantially reworked and extended before their submission to the present special issue. All submissions were peer-reviewed in accordance with the high standards of this Journal. We thank all the authors and all the reviewers for their invaluable contributions and dedicated work.

Moscow, April 2017

Lev Beklemishev 